\title{
DISCRIMINAÇÃO SIMPLES E PAREAMENTO AO MODELO COM ESTÍMULOS \\ BIDIMENSIONAIS EM MACACOS-PREGO INFANTES
}

\author{
SIMPLE DISCRIMINATION AND MATCHING-TO-SAMPLE WITH TWO-DIMENSIONAL \\ STIMULI IN INFANT CAPUCHIN MONKEYS
}

\author{
CARLOS B. A. SOUZA, \\ LIANE J. S. DAHÁS, \\ ROMARIZ S. BARROS \\ (UNIVERSIDADE FEDERAL DO PARÁ)
}

\begin{abstract}
RESUMO
Investigou-se a possibilidade de ensinar discriminações simples com mudanças sucessivas nas funções dos estímulos (DSMS) e matching-to-sample de identidade (IDMTS) com estímulos bidimensionais a dois macacos-prego (Sapajus sp.) infantes (M24 e M25) e experimentalmente ingênuos. O treino de DSMS alternou tentativas nas quais apenas um estímulo era apresentado (tentativa com estímulo único) com tentativas com dois ou mais estímulos apresentados simultaneamente. Foi feita uma redução gradual da probabilidade de reforçamento nas tentativas com estímulo único, aproximando esse treino ao de IDMTS. Foram necessárias 52 sessões de treino para M25 e 59 sessões para M24 até que o critério de aprendizagem fosse alcançado. O treino de IDMTS iniciou com dois modelos e duas comparações e continuou até atingir quadro modelos e quatro comparações. Foram necessárias 48 sessões com M24 e 51 com M25 para que as discriminações fossem aprendidas. Os resultados demonstraram que macacos-prego infantes podem adquirir ambos os repertórios. Os resultados são comparados com os de macacos-prego adultos nas mesmas tarefas e com os de macacos-prego adultos e infantes nas mesmas tarefas realizadas com objetos tridimensionais.
\end{abstract}

Palavras-chave: Discriminação simples; matching-to-sample de identidade; macaco-prego infante.

\begin{abstract}
This study investigated the possibility of teaching repeated shifts of simple discrimination (RSSD) and identity matching to sample (IDMTS) using two-dimensional stimuli in two experimentally naive infant capuchin monkeys (Sapajus sp.; subjects M24 and M25). RSSD training occurred by alternating trials in which only one stimulus was presented (single stimulus trial) with trials in which two or more stimuli were presented simultaneously. The reinforcement probability was gradually reduced in single-stimulus trials, making this training gradually more similar to the IDMTS procedure. Fifty-nine sessions were conducted with subject M24, and 52 sessions were conducted with subject M25 until the criterion was reached. The subjects then underwent IDMTS training, beginning with two choices and gradually moving to four choices. Forty-eight sessions were conducted with M24, and 50 sessions were conducted with M25 until the criterion was reached. The results showed that infant capuchins can acquire these repertoires. The present results were compared to previous studies that used the same kind of task in adult capuchin monkeys and compared to data obtained in the same task using three-dimensional objects as stimuli in adult and infant capuchins.

Keywords: Simple discrimination; identity matching-to-sample; infant capuchin monkeys

Trabalho realizado com apoio do CNPq (na forma de Bolsa de Produtividade em Pesquisa para Carlos Souza e Romariz Barros e de Iniciação Científica para Liane Dahás) e do Instituto Nacional de Ciência e Tecnologia sobre Comportamento, Cognição e Ensino INCT-ECCE (FAPESP / Processo \#2008/57705-8 - CNPq / Processo \#573972/2008-7).
\end{abstract}


O comportamento simbólico pode ser caracterizado, de maneira ampla, como o comportamento "controlado por relações entre estímulos dissimilares arbitrariamente relacionados (...) e substituíveis entre si” (Barros, Galvão, Brino, Goulart, \& McIlvane, 2005, p. 16). Dentro dessa ampla definição de comportamento simbólico, uma grande variedade de diferentes repertórios tem sido investigada, como, por exemplo, o responder (estabelecido convencionalmente) a relações arbitrárias entre sons/gestos/símbolos e estímulos ambientais (Horne, Lowe, \& Harris, 2007; SavageRumbaugh et al., 1993), repertórios de discriminação simples com reversões repetidas da função discriminativa dos estímulos - RRDS ${ }^{1}$ (D’Amato, Etkin, \& Fazzaro, 1968; Goulart, Galvão, \& Barros, 2003; Heise, 1959; Lavrati, 2002) e matching-tosample de identidade - IDMTS (Barros, Galvão, \& McIlvane, 2002; Goulart, Mendonça, Barros, Galvão, \& McIlvane, 2005; Souza, Borges, Goulart, Barros, \& Galvão, 2009).

Tem sido comum nos estudos sobre aquisição de repertórios simbólicos o uso de primatas nãohumanos (e.g., Martinez, \& Matsuzawa, 2009a, 2009b - para uma revisão ver Tomasello, \& Call, 1997). Como são evolutivamente/geneticamente próximos aos seres humanos, acredita-se que primatas podem fornecer modelos ideais para se estudar a evolução (e a dependência em relação à linguagem humana) de repertórios comportamentais comumente caracterizados como únicos do homem (Barros, Galvão, \& McIlvane, 2003; McIlvane et al., 2011) - e por vezes como provenientes da sua cultura (Tomasello, 2001).

Um gênero deprimatadonovomundo, omacacoprego (Sapajus sp.), apresenta comportamentos como a utilização de ferramentas e a aprendizagem por imitação (Fragaszy, Izar, Visalberghi, Ottoni, \& Oliveira, 2004; Moura \& Lee, 2004; Ottoni \& Izar, 2008), considerados indicadores de compreensão das relações entre eventos ambientais e comportamentos. A existência desses comportamentos na espécie a qualificou para ser utilizada como modelo animal em pesquisas relacionadas com a aquisição de repertórios simbólicos (Barros et al., 2003, McIlvane et al., 2011; Nagahama \& Souza, 2013).
Galvão, Barros, Rocha, Mendonça e Goulart (2002) e Barros, Galvão e Rocha (2005) sugeriram que ao menos rudimentos de comportamentos simbólicos podem ser produzidos nessa espécie, de maneira mais consistente, se esse repertório for sendo construído através de um programa de treino de discriminações gradualmente mais complexas, como numa espécie de curriculum. Nesse procedimento, a aprendizagem de tarefas mais simples poderia facilitar a realização de tarefas mais complexas. Por exemplo, o treino de RRDS, ao estabelecer a contingência de alterações nas funções de estímulos, presente no treino de IDMTS, poderia facilitar a aquisição deste repertório (Barros et al. 2002; Barros et al., 2005; Brandão, 2001; Galvão et al., 2005; Lavratti, 2002).

Barros (1998), Brandão (2001) e Lavratti (2002) apresentaram os primeiros dados reforçando a hipótese sobre a importância do treino de RRDS para um desempenho adequado em IDMTS. Esses estudos realizaram testes de identidade generalizada com macacos-prego, com e sem a história prévia de treino de RRDS com os estímulos do teste de IDMTS. Barros (1998) e Lavratti (2002) relataram que os resultados foram positivos nos dois testes de identidade generalizada cujos estímulos já eram conhecidos no contexto de RRDS, mas negativos quando eram completamente novos. No experimento de Brandão (2001), dos três testes com estímulos completamente novos, obteve-se resultado positivo em somente um deles; já os dois testes realizados com estímulos conhecidos (por terem sido usados nos treinos de RRDS) apresentaram resultados positivos. Portanto, estes dados sugeriam que o treino de RRDS poderia ser um facilitador da tarefa de IDMTS com os mesmos estímulos, mas não necessariamente um pré-requisito.

Lima et al. (2007), utilizando estímulos tridimensionais (diferentes objetos), observaram o mesmo tipo de efeito que os estudos de Barros (1998), Brandão (2001) e Lavratti (2002) de história de treino de RRDS sobre o desempenho em IDMTS e em testes de identidade generalizada com macacosprego. Os dois sujeitos do estudo passaram por testes de identidade generalizada com os mesmos três conjuntos de estímulos. Um dos sujeitos também foi submetido a um quarto conjunto de estímulos.

\footnotetext{
RRDS é um procedimento no qual dois estímulos são apresentados ao sujeito (ex. A1+ e A2-). Somente as respostes de escolha a um deles (A1+) é reforçada. Quando o sujeito responde consistentemente a esse estímulo, as funções de S+ e S- são revertidas (A2+/A1-), passando a ser reforçadas respostas apenas ao outro estímulo. Quando o desempenho se torna preciso é realizada outra reversão, e assim por diante.
} 
Um sujeito apresentou identidade generalizada em dois dos três testes realizados com um treino prévio de RRDS, mas nenhuma evidência desse repertório generalizado nos testes sem tal treino prévio. O segundo sujeito respondeu corretamente em um dos testes de identidade generalizada com estímulos completamente novos.

Deve-se ressaltar que os estudos descritos anteriormente foram todos realizados com macacosprego adultos. Considerando que a literatura sobre aquisição de comportamento simbólico tem sugerido a importância da exposição precoce às contingências ambientais como fator facilitador e até mesmo ampliador deste repertório (Deacon, 1997; Savage-Rumbaugh et al., 1993, Sousa, Okamoto, \& Matsuzawa, 2003; Tomasello, 2003), uma análise do desempenho de sujeitos infantes (com menos de dois anos de idade - Fragaszy, Visalberghi, \& Fedigan, 2004) nas tarefas de RRDS e IDMTS é uma questão pendente nessa linha de estudo.

Um primeiro estudo (Souza, Fonseca, \& Dahás, 2011) procurou confirmar o resultado de aquisição de discriminação simples com mudanças sucessivas da função de estímulos (DSMS) ${ }^{2}$ e IDMTS com estímulos tridimensionais por macacos-prego adultos (Lima et al., 2007), e avaliar a possibilidade de macacos-prego infantes aprenderem estes repertórios. Dois macacos-prego infantes e dois adultos passaram por um treino inicial de DSMS que alternou tentativas nas quais apenas um estímulo (o $\mathrm{S}+$ ) era apresentado com tentativas com dois ou mais estímulos apresentados simultaneamente. Foi feita uma redução gradual da probabilidade reforçamento nas tentativas com estímulo único, aproximando esse treino ao de IDMTS. O treino de IDMTS iniciou-se com dois modelos e duas comparações e prosseguiu até atingir quatro modelos e quatro comparações. Todos os sujeitos aprenderam as tarefas de DSMS e IDMTS.

O presente trabalho investigou a possibilidade de ensinar a macacos-prego infantes discriminações simples com mudanças sucessivas nas funções dos estímulos(DSMS) ematching-to-sampledeidentidade (IDMTS) utilizando estímulos bidimensionais, uma vez que essa modalidade de estímulos é a que tem sido utilizada na maioria dos estudos com macacos-prego adultos mencionados anteriormente (e.g., Barros, 1998; Brandão, 2001; Lavratti, 2002). Os resultados são comparados também com os de Lima et al., 2007 e de Souza et al. (2011).

\section{MÉTODO}

\section{Sujeitos}

Dois macacos-prego (Sapajus sp.), machos, experimentalmente ingênuos, com menos de um ano de idade no início do estudo, denominados M24 e M25. Os sujeitos foram mantidos em uma gaiolaviveiro junto com outros macacos-prego, alimentados duas vezes ao dia e com livre acesso a água. As condições de manutenção, manejo, alimentação e saúde, e os procedimentos experimentais foram aprovados pelo IBAMA e seguiram as normas da APA de condução ética no cuidado e uso de animais, tendo sido aprovadas junto ao Comitê de Ética em Pesquisa com Animais do Instituto de Ciências Biológicas da Universidade Federal do Pará.

\section{Equipamentos.}

Uma câmara experimental $(0,80$ x 0,80 x 0,70 m) com um lado fechado por material transparente e os demais lados feitos de tela metálica. Em um dos lados de metal havia uma porta de $0,25 \times 0,40$ $\mathrm{m}$ pela qual o sujeito era introduzido e retirado da gaiola. Na parede à direita da porta havia uma tela sensível ao toque de 15”. Acoplados à câmara, havia dois dispensadores automáticos de pelotas (Med Associates - Inc) que liberavam pelotas de alimento para macacos em um coletor localizado abaixo da tela. Um computador (Intel Pentium III com o aplicativo TREL V. 2.1, desenvolvido por José Iran Ataíde dos Santos) controlava a apresentação dos estímulos, registrava as respostas dos sujeitos e acionava o dispensador de pelota quando a resposta era correta (para maiores detalhes quanto ao equipamento, ver Soares Filho, 2010).

\section{Estímulos.}

Cinco figuras de cor preta feitas no programa Paint do Windows em um fundo branco quadrado de $2 \times 2 \mathrm{~cm}$ na tela sensível ao toque (ver Figura 1).

Quando são utilizados mais de dois estímulos o termo mais adequado para descrever a mudança da função dos estímulos é discriminações simples com mudanças sucessivas nas funções dos estímulos (DSMS) (ver Dahás, Brasiliense, Barros, Costa, \& Souza, 2010). 


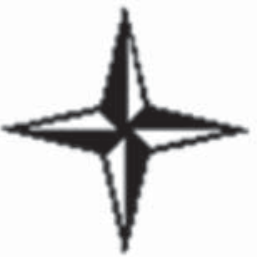

A1

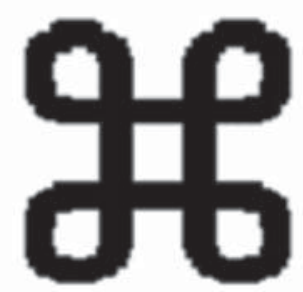

A2

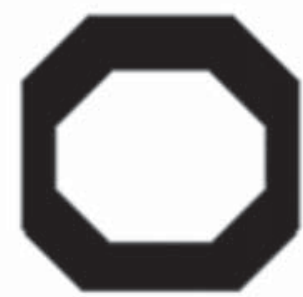

A3

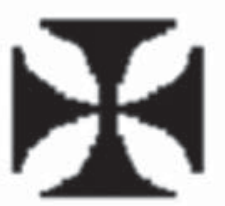

A4

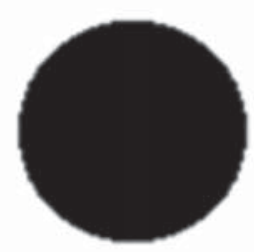

A5

Figura 1. Estímulos apresentados aos sujeitos na tela sensível ao toque. O estímulo A5 foi usado para substituir A4 (ver detalhes na seção de resultados).

\section{Procedimento.}

Em uma primeira fase, o comportamento dos sujeitos foi modelado até que fossem estabelecidas as respostas de entrar e sair da gaiola experimental e de tocar figuras na tela do computador. Depois disto, foi realizada a fase de treino de DSMS. Como esta fase tinha por objetivo facilitar a aquisição do repertório de IDMTS, e considerando que estudos anteriores mostravam alguns sujeitos que levavam diversas sessões para passar de uma fase a outra (Brandão, 2001; Goulart, Galvão, \& Barros, 2003; Lavratti, 2002), foi utilizado aqui um novo procedimento de discriminação simples, que alternava tentativas nas quais um único estímulo era apresentado (nomeadas de tentativas com estímulo único) e tentativas nas quais dois ou mais estímulos eram apresentados (nomeadas de tentativas de escolha), sendo um deles (o $\mathrm{S}+$ ) o mesmo da tentativa com estímulo único imediatamente anterior - ver Treino de DSMS a seguir. Como essa apresentação de estímulos se assemelha àquela da situação de pareamento ao modelo (visto que há primeiramente a apresentação de um estímulo idêntico ao que deve ser escolhido na tentativa), acreditava-se que tal semelhança facilitasse a passagem para o treino de IDMTS (ver Rico, 2006). Ambos os tipos de tarefas foram treinadas em uma ou duas sessões por dia, de segunda a sexta-feira.

Treino de discriminações simples com mudanças sucessivas nas funções dos estímulos (DSMS). Neste treino, um estímulo com função de S+ era apresentado inicialmente sozinho na tela do computador (tentativa com estímulo único) e a resposta de tocá-lo era reforçada (posteriormente o reforçamento dessa resposta era reduzido a ter ser eliminado - ver descrição a seguir). Imediatamente após essa resposta, o mesmo estímulo era apresentado, novamente como $\mathrm{S}+$, mas agora simultaneamente com outro estímulo com função de S- (tentativa de escolha - cada estímulo podia ocupar uma de nove possíveis posições em uma matriz de 3x3 na tela ver Barros et al., 2002). Respostas ao S+ produziam reforçamento e um Intervalo Entre Tentativas (IET) de 6s (produzido também por uma resposta ao S-) antes da apresentação de uma nova tentativa com estímulo único com o mesmo S+.

Como apresentado na Tabela 1 , as primeiras sessões de treino de DSMS foram de 20 tentativas com um par de estímulos (A1+, A2-), com critério de aprendizagem de $90 \%$ de acertos. Após a $12^{\mathrm{a}}$ sessão (para M24) e a 11 a (para M25), o critério de aprendizagem foi alterado para oito respostas corretas consecutivas, em sessões de 40 tentativas possíveis (com a finalidade de possibilitar um número maior de tentativas de DSMS). Uma vez que o sujeito atingia o critério de aprendizagem, na próxima sessão as funções dos estímulos eram revertidas (Etapa 2). Depois de ocorrida a primeira reversão e de os sujeitos alcançarem o critério para outra reversão, era acrescentado, nas tentativas de escolha, um terceiro estímulo, com função negativa (Etapa 3). Assim que o sujeito atingisse o critério, ocorria uma mudança na função dos estímulos, com aquele recentemente introduzido passando a funcionar como S+ (Etapa 4). Quando o critério era alcançado novamente, uma nova mudança era feita, sendo que o único estímulo que ainda não tinha funcionado como $\mathrm{S}+$ na presença do estímulo novo passava então a funcionar como S+ (Etapa 5). Após o alcance de critério nessa etapa, um quarto estímulo era apresentado nas tentativas de escolha, e o procedimento anterior se repetia: com o acréscimo do novo estímulo, o estímulo anteriormente positivo continuava sendo o $\mathrm{S}+$ (Etapa 6), depois a função positiva era alterada para o estímulo novo (Etapa 7), e posteriormente para os outros dois estímulos (Etapas 8 e 9) (ver Tabela 1).

A partir da sétima mudança na função dos estímulos (Etapa 10), o reforçamento das repostas nas tentativas com estímulo único foi reduzido 
para $50 \%$, o que se manteve para a Etapa 11. Essa etapa foi então novamente realizada, reforçando-se somente as cinco primeiras tentativas com estímulo único de cada sessão. Alcançado o critério para a próxima mudança (Etapa 12), manteve-se o reforço apenas para as cinco primeiras tentativas com estímulo único de cada sessão. Uma vez alcançado o critério de aprendizagem, esta Etapa foi repetida com reforço apenas para as três primeiras tentativas com estímulo único de cada sessão até o critério de aprendizagem para a próxima mudança (Etapa13). Nesta etapa, primeiro foi mantido o reforço apenas para as três primeiras tentativas com estímulo único. Depois de atingido o critério de aprendizagem, a etapa foi repetida sem reforço nas tentativas com estímulo único. Quando o critério de aprendizagem foi alcançado novamente, foram realizadas mais duas mudanças nas funções dos estímulos (Etapas 14 e 15) sem reforço nas tentativas com estímulo único (ver Tabela 1). Alcançado o critério de aprendizagem na Etapa 15, tinha início o treino de IDMTS. Esperavase que estas sessões sem reforço nas tentativas com estímulo único, devido à semelhança de configuração com o treino de IDMTS, facilitassem a transição para este tipo de treino.

Treino de matching-to-sample de identidade (IDMTS). A configuração dos estímulos neste treino era semelhante à configuração das sessões do treino de DSMS nas quais as respostas nas tentativas com estímulo único não eram reforçadas: um estímulo era apresentado na tela do computador (Estímulo Modelo) e a resposta de tocá-lo produzia imediatamente o seu desaparecimento e o surgimento imediato dos estímulos comparação (o mesmo estímulo como S+ e outro[s] como S-). A cada tentativa, os estímulos ocupavam uma das nove possíveis posições na matriz de $3 \times 3$ na tela do computador. Respostas ao S+ produziam reforçamento e o IET. Respostas ao S- produziam apenas o IET. Em ambos os casos, seguia-se a apresentação de uma nova tentativa, que podia ser com o mesmo estímulo modelo da tentativa anterior ou outro, como será detalhado a seguir. As sessões foram de 40 ou 36 tentativas.

Como demonstra a Tabela 2, esse treino foi realizado inicialmente com sessões em que o mesmo estímulo (A1 ou A2) era apresentado como modelo nas 20 primeiras tentativas, e outro estímulo (A1 ou A2) como modelo nas 20 últimas tentativas da mesma sessão, tendo apenas os dois estímulos - A1 e A2 como comparações (Etapa 1), de forma a possibilitar um aumento gradual na complexidade discriminativa exigida na tarefa.

O critério de aprendizagem era de $80 \%$ de acerto em cada um desses dois tipos de tentativas, critério mantido no restante do treino de IDMTS. Após o critério ser atingido, o procedimento foi repetido com os outros dois estímulos (Etapa 2). Em seguida, iniciou-se o treino com quatro estímulos, aparecendo cada um como modelo, mas somente dois como comparações. Primeiro foram realizadas sessões com 10 tentativas consecutivas para cada modelo (Etapa 3). Após o critério, passou-se para sessões de oito blocos de cinco tentativas consecutivas (Etapa 4), e depois para blocos de cinco (primeiro) e de duas tentativas consecutivas com o mesmo modelo (em um total de 40 tentativas) (Etapa 5). Em seguida foram realizadas sessões nas quais as 10primeiras tentativas eram de blocos de duas tentativas, e as restantes 30 eram randomizada (Etapa 6). Após o critério nessa etapa, foram realizadas sessões totalmente randomizadas com quatro estímulos como possíveis modelos, tendo inicialmente três comparações (Etapa 7), e após o critério em sessões com quatro comparações (Etapa 8). As Etapas 7 e 8 foram realizadas com 36 tentativas por sessão (ver Tabela 2).

\section{REsultados}

A Tabela 3 mostra os resultados do treino de DSMS com os dois sujeitos. Como o número de tentativas necessárias para cada etapa foi relativamente proporcional ao número de sessões, foram tomados apenas estes últimos para análise. Foram realizadas 59 e 52 sessões respectivamente com os sujeitos M24 e M25 até que fosse concluído o treino de DSMS em suas 15 etapas. O desempenho dos sujeitos alcançou o critério após a primeira mudança em oito (M24) e 10(M25) sessões (Etapa 2). Já com a adição do segundo estímulo como S-, sem que fosse realizada mudança, o critério de precisão foi alcançado em uma única sessão para cada sujeito (Etapa 3). Após a segunda mudança, foram realizadas cinco (M24) e três (M25) sessões para o alcance do critério (Etapa 4), e após a terceira, oito (M24) e três (M25) sessões (Etapa 5). Com a adição da quarta comparação, para ambos os sujeitos, o critério foi alcançado em uma única sessão (Etapa 6). A quarta mudança foi realizada em quatro sessões com ambos os sujeitos (Etapa 7), e a quinta em três sessões (Etapa 8). Após a sexta mudança foram necessárias sete sessões para M24 e quatro para M25 atingirem o critério de aprendizagem (Etapa 9). 
Tabela 1. Etapas do treino de DSMS

\begin{tabular}{|c|c|c|c|c|c|c|c|c|}
\hline \multirow[t]{2}{*}{ Etapas } & \multirow{2}{*}{$\begin{array}{l}\text { Estímulo } \\
\text { único }\end{array}$} & \multirow{2}{*}{$\begin{array}{l}\text { Reforçamento da resposta } \\
\text { ao estímulo único }\end{array}$} & \multicolumn{4}{|c|}{$\begin{array}{l}\text { Estímulos apresentados em } \\
\text { tentativa de escolha }\end{array}$} & \multirow[t]{2}{*}{ Critério } & \multirow{2}{*}{$\begin{array}{c}\text { Total de } \\
\text { tentativas na } \\
\text { sessão }\end{array}$} \\
\hline & & & $\mathrm{S}+$ & S- & S- & S- & & \\
\hline 1 & A1 & & A1 & $\mathrm{A} 2$ & - & - & $90 \%$ & 20 \\
\hline 2 & A2 & & A2 & A1 & - & - & $90 \%$ & 20 \\
\hline 3 & A2 & & A2 & A1 & A3 & - & & 20 \\
\hline 4 & A3 & & A3 & A1 & $\mathrm{A} 2$ & - & & \\
\hline 5 & A1 & $100 \%$ & A1 & A2 & A3 & - & & \\
\hline 6 & A1 & & A1 & $\mathrm{A} 2$ & A3 & A4 & & \\
\hline 7 & A4 & & A4 & A1 & A2 & A3 & & \\
\hline 8 & A3 & & A3 & A1 & $\mathrm{A} 2$ & A4 & & \\
\hline 9 & A2 & & A2 & A1 & A3 & A4 & $8 \mathrm{CC}$ & 40 \\
\hline 10 & A1 & $50 \%$ & A1 & A2 & A3 & A4 & & \\
\hline 11 & A4 & $50 \% / 5 p t$ & A4 & A1 & $\mathrm{A} 2$ & A3 & & \\
\hline 12 & A3 & $5 p t / 3 p t$ & A3 & A1 & A2 & A4 & & \\
\hline 13 & A2 & $3 p t / 0$ & $\mathrm{~A} 2$ & A1 & A3 & A4 & & \\
\hline 14 & A1 & 0 & A1 & A1 & A2 & A3 & & \\
\hline 15 & A4 & 0 & A4 & A1 & A2 & A3 & & \\
\hline
\end{tabular}

8CC= oito respostas corretas consecutivas em tentativas de escolha. 5pt e 3pt= Cinco e três primeiras tentativas com estímulo único da sessão. Ver texto para explicação mais detalhada.

Tabela 2. Etapas do treino de IDMTS

\begin{tabular}{ccccc}
\hline Etapa & $\begin{array}{c}\text { N }^{\text {de }} \\
\text { Comparações }\end{array}$ & Modelos & Tentativas & Agrupamento de Tentativas \\
\hline 1 & 2 & A1 A2 & 40 & 2 blocos de 20 tentativas consecutivas \\
2 & 2 & A3 A4 & 40 & 2 blocos de 20 tentativas consecutivas \\
3 & 2 & A1 A2 A3 A4 & 40 & 4 blocos de 10 tentativas consecutivas \\
4 & 2 & A1 A2 A3 A4 & 40 & 8 blocos de 5 tentativas consecutivas \\
5 & 2 & A1 A2 A3 A4 & 40 & Blocos de 5 e 2 tentativas consecutivas \\
6 & 2 & A1 A2 A3 A4 & 40 & Randomizadas após a 10 ${ }^{\text {a tentativa }}$ \\
7 & 3 & A1 A2 A3 A4 & 36 & Randomizadas \\
8 & 4 & A1 A2 A3 A4 & 36 & Randomizadas \\
\hline
\end{tabular}

Com o início da redução na probabilidade de reforçamento nas tentativas com estímulo único, ambos os sujeitos alcançaram o critério após a sétima mudança em cinco sessões (Etapa 10) e após a oitava mudança em duas sessões (Etapa 11). Após a nona mudança em duas (M24) e sete (M25) sessões (Etapa 12). Para a 10 ${ }^{\text {a }}$ (Etapa 13$), 11^{\text {a }}$ (Etapa 14) e $12^{\mathrm{a}}$ mudanças (Etapa 15) M24 necessitou, respectivamente, de três, uma e três sessões para atingir o critério, enquanto M25 necessitou de uma, uma e duas sessões (as duas últimas mudanças já sem reforçamento nas tentativas com estímulo único).

A Tabela 4 exibe os dados dos treinos de IDMTS com os sujeitos M24 e M25, que realizaram um total de 48 e 51 sessões, respectivamente, no decorrer das oito Etapas de treino dessa fase. Ambos os sujeitos alcançaram o critério de aprendizagem em seis sessões na Etapa de dois blocos de 20 tentativas consecutivas (Etapas 1 e 2); em duas sessões na Etapa de quatro blocos de 10 tentativas consecutivas (Etapa 3); em uma sessão nas Etapas de oito blocos de cinco tentativas consecutivas (Etapa 4) e de blocos de cinco e duas tentativas consecutivas (Etapa 5). Na Etapa 6, de tentativas randomizadas após a $10^{\mathrm{a}}$ tentativa M24 necessitou de duas sessões e M25 de quatro sessões. Após esta Etapa, foi acrescentada uma terceira comparação, e os sujeitos levaram 18 (M24) e 22 (M25) sessões até o critério (Etapa 7). Com o acréscimo da quarta comparação (Etapa 8), observou-se que o sujeito M25 passou a errar de forma sistemática na escolha do estímulo A4 (dado não apresentado). Em função disto, a partir da $15^{\mathrm{a}}$ sessão para M24 e $13^{\mathrm{a}}$ para M25 houve a troca do estímulo A4 pelo A5 e a adoção de um procedimento de correção que consistia em repetira tentativa até que a resposta correta fosse emitida (essas adaptações do 
procedimento foram realizadas também com o sujeito M24 procurando garantir a comparabilidade dos resultados obtidos). Após essas alterações os sujeitos alcançaram o critério para o término do treino em 18 (M24) e 15 (M25) sessões.

\section{DISCUSSÃO}

Os dados deste estudo mostram a aquisição de discriminações simples (com mudanças repetidas das funções dos estímulos) e matching-to-sample de identidade com estímulos bidimensionais em macacos-prego infantes. Junto com os dados de Souza et al. (aceito), que mostrou a aquisição dos mesmos repertórios com estímulos tridimensionais (objetos) por outros dois macacos-prego infantes, estes resultados mostram que repertórios considerados relevantes para a aquisição de comportamentos complexos (e.g.. identidade generalizada e discriminações condicionais arbitrárias) podem ser aprendidos nos períodos iniciais de desenvolvimento biocomportamental desta espécie.

Os infantes do presente estudo apresentaram aprendizagem mais rápida dos repertórios de DSMS e IDMTS quando comparados com outros estudos com macacos-prego adultos (ver Barros, 1998; Barros et al., 2002; Brandão, 2001; Lavratti, 2002). Outra diferença foi quanto à randomização das respostas: os macacos-prego adultos apresentavam grande persistência comportamental (Dube, \& McIlvane, 2002) ao escolher o estímulo correlacionado com o reforçamento após a reversão da função do estímulo, e o faziam por muitas sessões (Brandão, 2001; Lavratti, 2002). Isso não ocorreu com os sujeitos deste estudo, que mostraram relativamente baixa resistência às mudanças nas contingências, passando a responder ao novo estímulo $\mathrm{S}+$ em poucas tentativas.

Fragaszy, Visalberghi, et al. (2004) indicam que macacos-prego, comparativamente a outros primatas não humanos (com exceção de chimpanzés), apresentam maior desenvolvimento cerebral após o nascimento, o que aumenta a possibilidade das suas interações com o ambiente influenciarem no seu desenvolvimento neuronal. Essa característica do desenvolvimento dos macacos-prego poderia ser um fator facilitador para a aprendizagem de novos repertórios nos infantes.

No entanto, deve-se levar em consideração o fato de que no presente estudo foi utilizado o procedimento de DSMS com tentativas com estímulo único. Este procedimento pode ter tido influência nas diferenças de desempenho e padrão de respostas descritas anteriormente. Portanto, estudos posteriores comparando o desempenho de infantes no mesmo procedimento e o desempenho destes com adultos no mesmo procedimento e em outros (e.g., treino clássico de DSMS), deverão analisar se o desempenho de infantes e adultos está relacionado ao procedimento (que pode ajudar a prevenir a ocorrência de persistência comportamental - ver Rico, 2006) ou a maior variabilidade comportamental presente no comportamento de infantes ainda sem história de reforçamento.

Acredita-se que a utilização do procedimento de DSMS com tentativas com estímulo único, por se assemelhar, do ponto de vista das contingências estabelecidas, a tentativas de IDMTS (já que apresenta um "modelo" fidedigno de qual estímulo será o S+ na próxima tentativa), pode ser de grande valia para a rápida aquisição do repertório de IDMTS. Apesar de ainda se fazer necessário o teste dessa hipótese com sujeitos ingênuos sendo treinados em um procedimento clássico de DSMS, sem as tentativas com estímulo único, pode-se afirmar que os estudos que até o presente momento utilizaram o procedimento com tentativas com estímulo único apresentaram um número menor de sessões (Rico, 2006; Souza et al., 2011) em comparação com estudos anteriores realizados com treinos de DSMS sem as tentativas com estímulo único (Barros et al., 2002; Lima et al., 2007).

Os resultados de dois macacos-prego infantes e de um adulto (M27) no estudo de Souza et al. (2011), mostraram que o mesmo procedimento do presente estudo realizado com objetos (estímulos tridimensionais) implicou em um número maior de sessões para a aquisição de DSMS (80 e 94 para os infantes e 103 para o adulto). Por outro lado, foi documentada aquisição relativamente rápida das discriminações no treino com estímulos tridimensionais com um dos macacos-prego adulto (M18) do estudo de Souza et al. (22 sessões para o critério de DSMS) e outro (M13) do estudo de Lima et al. (2007) (menos de 20 sessões para o critério mas neste último o treino foi realizado apenas com dois estímulos, com um critério de quatro reversões sucessivas). No entanto, ambos os macacos-prego adultos tinham histórias prévias de treino de DSMS e IDMTS com estímulos bidimensionais, o que sugere um papel importante da história prévia de exposição às contingências das tarefas. 
Tabela 3. Número de tentativas e de sessões até o critério em cada Etapa do treino de DSMS para M24 e M25

\begin{tabular}{|c|c|c|c|c|c|c|c|}
\hline \multicolumn{4}{|c|}{ Estímulos } & \multicolumn{2}{|c|}{ Tentativas } & \multicolumn{2}{|c|}{ Sessões } \\
\hline$S+$ & \multirow{2}{*}{\multicolumn{2}{|c|}{ A2 }} & & M24 & M25 & M24 & M25 \\
\hline A1 & & & & 120 & 100 & 6 & 5 \\
\hline A2 & A1 & - & - & 171 & 228 & $8^{*}$ & $10^{*}$ \\
\hline A2 & A1 & A3 & - & 12 & 8 & 1 & 1 \\
\hline A3 & A1 & A2 & - & 168 & 92 & 5 & 3 \\
\hline A1 & A2 & A3 & - & 317 & 94 & 8 & 3 \\
\hline A1 & A2 & A3 & A4 & 14 & 21 & 1 & 1 \\
\hline A4 & A2 & A3 & A1 & 141 & 153 & 4 & 4 \\
\hline A3 & A2 & A1 & A4 & 102 & 88 & 3 & 3 \\
\hline A2 & A1 & A3 & A4 & 256 & 142 & 7 & 4 \\
\hline A1 & A2 & A3 & A4 & 193 & 168 & 5 & 5 \\
\hline A4 & A1 & A2 & A3 & 62 & 71 & 2 & 2 \\
\hline A3 & A1 & A2 & A4 & 60 & 204 & 2 & 7 \\
\hline A2 & A1 & A3 & A4 & 94 & 16 & 3 & 1 \\
\hline A1 & A2 & A3 & A4 & 37 & 16 & 1 & 1 \\
\hline A4 & $\mathrm{A} 2$ & A3 & A1 & 92 & 48 & 3 & 2 \\
\hline & TOT & & & 1839 & 1449 & 59 & 52 \\
\hline
\end{tabular}

* Após a sexta sessão, modificou-se o critério de 90\% de acerto para o de oito corretas consecutivas (ver Procedimento)

Tabela 4. Número de sessões no treino de IDMTS para M24 e M25

\begin{tabular}{clcc}
\hline \multirow{2}{*}{ Número de Comparações } & \multicolumn{1}{c}{ Modelos } & M24 & M25 \\
\hline 2 & A1, A2 & 3 & 3 \\
2 & A3, A4 & 3 & 3 \\
2 & A1, A2, A3, A4 & 2 & 2 \\
2 & A1, A2, A3, A4 & 1 & 1 \\
2 & A1, A2, A3, A4 & 1 & 1 \\
2 & A1, A2, A3, A4 & 2 & 4 \\
3 & A1, A2, A3 A4 & 18 & 22 \\
4 & A1, A2, A3, A4/A5 & & $18^{*}$ \\
\hline
\end{tabular}

* Correção a partir da $15^{\mathrm{a}}$ sessão.

** Correção a partir da $13^{\mathrm{a}}$ sessão.

A comparação dos resultados do presente estudo com o estudo de Souza et al. (2011) sugere que há 1) uma maior facilidade de sujeitos ingênuos na aprendizagem de DSMS com estímulos bidimensionais; e 2) um efeito facilitador da história prévia de exposição às contingências, ainda que com estímulos em outra modalidade perceptual. Novos estudos devem procurar avaliar de maneira mais precisa o desempenho nos treino de DSMS e IDMTS comparando infantes vs. adultos, estímulos bidimensionais vs. tridimensionais, sujeitos ingênuos vs. com história experimental (intra eintermodalidades perceptuais).

\section{REFERÊNCIAS}

Barros, R. S. (1998). Controle do comportamento por relações entre estímulos. Tese de Doutorado. Programa de Pós Graduação em Psicologia Experimental. Universidade de São Paulo.

Barros, R. S., Galvão, O. F., \& McIlvane, W. (2002). Generalized identity matching-to sample in Cebus apella. The Psychological Record, 52, 441 460.

Barros, R. S., Galvão, O. F., \& McIlvane, W. (2003). The search for relational learning capacity in Cebus apella: A programmed "educational" approach. Em S. Soraci Jr \& K. Murata -Soraci (Eds.), Visual information processing (pp. 223 245). Westport: Praeger. 
Barros, R. S., Galvão, O. F., \& Rocha, A. C. (2005). O pesquisador na escola experimental de primatas: de experimentador a programador de contingências. Interação em Psicologia, 9, 201 214.

Barros, R. S., Galvão, O. F., Brino, A. L. F., Goulart, P. R. K., \& McIlvane, W. J. (2005). Variáveis de procedimento na pesquisa sobre classes de equivalência: contribuições para o estudo de comportamento simbólico. Revista Brasileira de Análise do Comportamento, 1, 15 27.

Brandão, S. (2001). Programa de ensino de escolha de acordo com o modelo por identidade generalizada a partir de discriminações simples com Cebus apella. Dissertação de Mestrado. Universidade Federal do Pará, Belém do Pará.

D’Amato, M. R., Etkin, M., \& Fazzaro, J. (1968). Cueproducing behavior in the capuchin monkey during reversal, extinction, acquisition, and overtraining. Journal of the Experimental Analysis of Behavior, 11, 425 433.

Dahás, L. J. S., Brasiliense, I. C., Barros, R. S., Costa, T. D., \& Souza, C. B. A (2010). Formação de classes funcionais em cães domésticos (Canis familiaris): Uma abordagem da aquisição de comportamento pré simbólico. Acta Comportamentalia, 18, 317 346.

Deacon, T. W. (1997). Outside the brain. Em T. W. Deacon, The symbolic species: The corevolution of language and the brain. (pp.102 142). New York: Norton.

Dube, W. V., \& McIlvane, W. J. (2002). Reinforcer rate and stimulus control in discrimination reversal learning. The Psychological Record, 52, 405 416.

Fragaszy, D. M., Izar, P., Visalberghi, E., Ottoni, E. B., \& Oliveira, M. G. (2004). Wild capuchin monkeys (Cebus libidinosus) use anvils and stone pounding tools. American Journal of Primatology, 64, 359 366.

Fragaszy, D. M., Visalberghi, E., \& Fedigan, L. M. (2004). The complete capuchin: The biology of the genus Cebus. Cambridge: Cambridge University Press.

Galvão, O. F., Barros, R. S., Rocha, A. C., Mendonça, M., \& Goulart, P. R. K. (2002). Escola Experimental de Primatas. Estudos de Psicologia, 7, 361 370.

Galvão, O. F., Barros, R. S., Santos, J. R., Brino, A. L. F., Brandão, S., Lavratti, C. M., Dube, W. V., \& McIlvane, W. J. (2005). Extend limits of the matching concept in Cebus Apella: A matter of experimental control? The Psychological Record, 55, 219 232.

Goulart, P. R. K., Galvão, O. F., \& Barros, R. S. (2003). Busca de formação de classes de estímulos via procedimento de reversões repetidas de discriminação simples combinadas em macacos prego (Cebus apella). Interação em Psicologia,7, 109 120.
Goulart, P. R. K., Mendonça, M. B., Barros, R. S., Galvão, O. F., \& McIlvane, W. J. (2005). A note on select and reject -controlling relations in the simple discrimination of capuchin monkeys (Cebus apella). Behavioural Processes, 69, 295 302.

Heise, G. A. (1959). Discrimination reversal as a baseline for studying the acquisition of behavior. Journal of the Experimental Analysis of Behavior, 2, 251.

Horne, P. J., Lowe, C. F., \& Harris, F. D. A. (2007). Naming and categorization in young children: V. Manual sign training. Journal of the Experimental Analysis of Behavior, 87, 367 381.

Lavratti, C. M. (2002). Condições suficientes (e talvez necessárias) para a emergência de pareamento ao modelo por identidade generalizada em Cebus apella Dissertação de Mestrado. Universidade Federal do Pará, Belém do Pará.

Lima, M. E. A. C., Barros, R. S., Dahás, L. J. S., Cruz, A. P. C., Bezerra, D. S., \& Galvão, O. F. (2007). Discriminação simples e pareamento ao modelo por identidade com estímulos tridimensionais em macacos prego (Cebus apella). Acta Comportamentalia, 15, 5 20.

Martinez, L., \& Matsuzawa, T. (2009a). Auditory visual intermodal matching based on individual recognition in a chimpanzee (Pan troglodytes). Animal Cognition, 12, S71 S85.

Martinez, L., \& Matsuzawa, T. (2009b). Effect of species specificity in auditory visual intermodal matching in a chimpanzee (Pan troglodytes) and humans. Behavioural Processes, 82, 160 163.

McIlvane, W. J., Dube, W. V., Serna, R. W., Lionello DeNolf, K. M., Barros, R. S., \& Galvão, O. F. (2011). Some current dimensions of Translational Behavior Analysis: From laboratory research to intervention for persons with autism spectrum disorders. Em E. A. Mayville \& J. A. Mulick (Orgs.), Behavioral foundations of effective autism treatment (pp.155 181). Cornwall on Hudson, NY: Sloan.

Moura, A. C. A., \& Lee, P. C. (2004). Capuchin stone tool use in caatinga dry forest. Science, 306, 1909.

Nagahama, M. M., \& Souza, C. B. A. (2013). Escola Experimental de Primatas: Análise da coerência entre pressupostos e práticas empíricas. Revista Brasileira de Terapia Comportamental e Cognitiva.

Ottoni, E. B., \& Izar, P. (2008). Capuchin monkey tool use: Overview and implications. Evolutionary Anthropology, 17, 171-178.

Rico, V. V. (2006). Persistência comportamental e topografía de controle de estímulos coerente em treino de discriminação simples e escolha condicional 
por identidade ao modelo com quatro escolhas em macaco prego (Cebus apella) Dissertação de Mestrado. Universidade Federal do Pará, Belém do Pará.

Savage-Rumbaugh, E., Murphy, J., Sevick, R., Brakke, K., Williams, S., \& Rumbaugh, D. (1993). Language comprehension in ape and child. Monographs of the Society for Research in Child Development, 58, 1 256.

Soares Filho, P. S. D. (2010). Aquisição de relações condicionais simétricas e não simétricas e formação de classes por Cebus apella Dissertação de Mestrado. Universidade Federal do Pará, Belém do Pará.

Sousa, C., Okamoto, S., \& Matsuzawa, T. (2003). Behavioural development in a matching to sample task and token use by an infant chimpanzee reared by his mother. Animal Cognition, 6, 259 267.

Souza, C. B. A., Borges, R. P., Goulart, P. R. K., Barros, R. S., \& Galvão, O. F. (2009). Testes de identidade generalizada com objetos em macaco prego (Cebus apella). Psicologia: Teoria e Pesquisa, 25, 169 177.

Souza, C. B. A., Fonseca, A. R., \& Dahás, L. J. S. (2011). Discriminação simples e pareamento ao modelo com objetos em macacos prego. Revista Brasileira de Análise do Comportamento, 7, 1 10.

Tomasello, M. (2001). The cultural origins of human cognition. London: Harvard University Press.

Tomasello, M. (2003). Biological, cultural, and ontogenetic processes. Em M. Tomasello, Constructing a language. A used based theory of language acquisition. (pp.282 322). Cambrigde, MA: Harvard University Press.

Tomasello, M., \& Call, J. (1997). Primate cognition. New York: Oxford University Press. 